\title{
LITERACIDAD Y ÉTICA. PERCEPCIONES DE ESTUDIANTES DE EDUCACIÓN SUPERIOR SOBRE EL CURSO DE ÉTICA PROFESIONAL DENTRO DE LA CURRICULA
}

\author{
Literacy and Ethics: Perceptions of students of higher education regarding the \\ cours of Professional ethics within the curriculum
}

\section{César Correa Arias}

Fecha de recepción: 21/03/2019

Fecha de aceptación: 16/12/2019

RESUMEN: Esta investigación en proceso forma parte del proyecto de "Formación Docente, Pensamiento Crítico y Dialogicidad" de la Red Iberoamericana de Oralidad, adscrito en el Consejo Latinoamericano de Ciencias Sociales (CLACSO). Se analizaron las relaciones entre Literacidad y Ética desde una perspectiva socio-crítica, destacando los procesos de formación ético-política de los estudiantes de pregrado en México, en el horizonte del cultivo de la humanidad. Se estudiaron las percepciones de los estudiantes del centro universitario de ciencias económico-administrativas, de la Universidad de Guadalajara, México, acerca del papel de la enseñanza de la ética dentro del desarrollo de sus trayectos de formación y vida profesional, para identificar los obstáculos y oportunidades del acceso a fuentes y recursos de literacidad que permitan la construcción de una ciudadanía autónoma y responsable. La presente investigación se sitúa en un paradigma socio-crítico, utiliza un sub-paradigma éticoformativo-explorativo y emplea una metodología cualitativa. Se realizaron entrevistas a profundidad y grupos focales. Se recurrió al software Atlas.ti y se realizó un análisis crítico de discurso como técnicas para la sistematización y el análisis de datos respectivamente. Los resultados preliminares muestran una valoración significativa de las experiencias y conocimientos adquiridos por los estudiantes en los cursos de ética y una reacción contraria con respecto a la gran mayoría de cursos en la Universidad de Guadalajara. Mientras los cursos de ética favorecen los procesos de subjetividad e intersubjetividad, los demás cursos están centrados en contenidos programáticos que invisibilizan a los estudiantes.

PALABRAS CLAVE: educación superior, formación, literacidad, ética, ciudadanía global

ABSTRACT: This paper is part of a project called: "Teacher training, Critical Thinking, and Dialogicity", within the Ibero-American Network of Studies of Orality, and in the framework of the Latin American Council of Social Sciences (CLACSO). This research analyzed the relationships between Literacy and Ethics in a socio-critical perspective, emphasizing the processes of the ethical-political education of students in higher education in Mexico, in the horizon of cultivating humanity. We explored students' perceptions regarding the courses of Professional Ethics in the campus of Sciences of Economics and Management, University of Guadalajara, Mexico as well as the role ethics has in the development of the students' educational and professional trajectories. The main aim was to identify the obstacles and opportunities of access to sources of literacy for building autonomous and responsible citizenship. The research used a socio-critical paradigm, an educational-exploratory subparadigm, and a qualitative methodology. We used in-depth interviews and focus groups, and employed software Atlas.T for data organization and critical discourse analysis. Preliminary 
results show a significant recognition of the meaningful experiences and knowledge acquired by students in Ethics courses. However, the opposite reaction was found regarding most courses at the University of Guadalajara. While the Ethics courses favour the processes of subjectivity and inter-subjectivity, other courses are focused on programmatic contents that make students invisible. citizenship.

KEY WORDS: higher education, teacher education, literacy, ethics, global

\section{Introducción}

La literacidad, comprendida en la contemporaneidad como la adquisición de conocimientos que posibilitan una postura política y la construcción de la ciudadanía, recuerdan los componentes que conforman la conditio sine qua non de la constitución de la polis griega. La literacidad consiste en un sistema de capacidades humanas de adquisición y manejo de signos y símbolos que mediados por el leguaje permiten elaborar textos y discursos, diálogos y narrativas y, por tanto, de comunicar la experiencia humana. Igualmente, representa el fomento de un pensamiento complejo, político y crítico que promuevan el desarrollo de la subjetividad y la intersubjetividad, al igual que las capacidades humanas del juicio, la razón, la elección, la autonomía, la construcción moral y ética, entre otras.

Por su parte, la ética, concepto central en la edificación de la polis griega helénica, ha sido considerada como el fundamento de la justicia social, de los procesos de construcción de conciencia social y base del reconocimiento mutuo en instituciones justas (Ricoeur, 2006). La ética vincula la phrónesis y la sophia, una sabiduría práctica y un conocimiento reflexivo respectivamente, conceptos estructurales de la construcción de la comunidad en el mundo griego. No obstante, en el mundo moderno estos dos conceptos se han distanciado, al punto de lograr una fragmentación entre conocimiento y experiencia ético-política. En consecuencia, la conjunción entre literacidad y ética se presenta como una necesidad urgente para el conocimiento social de nuestras sociedades contemporáneas limitadas por el consumismo salvaje y la barbarie de la violencia, la impunidad, la corrupción y la indiferencia.

La investigación se desarrolló en su primera fase, analizando las percepciones de estudiantes de educación superior en México, particularmente en el Centro Universitario de Ciencias Económico-Administrativas de la Universidad de Guadalajara, en el período 20152018, acerca del papel que cumple la enseñanza de la ética dentro de sus trayectos de formación y vida profesional, a fin de identificar los obstáculos y oportunidades del acceso a fuentes y recursos de literacidad que permitan la construcción de una ciudadanía autónoma y responsable.

\section{Fundamentación teórica}

\subsection{La Literacidad y la estructuración de un pensamiento crítico}

La literacidad contiene orígenes históricos, culturales, lingüísticos, políticos y educativos heterogéneos. Sin embargo, tres de sus fuentes, una de carácter histórico, otra de carácter cognitivo y una de carácter ético, fueron de mayor interés en este estudio: 1) El 
desarrollo físico-intelectual de los gimnasios en Grecia helenística; 2) La enseñanza de las Tres erres (3Rs) Escribir, leer y contar como base cognitiva; y 3) Experiencia, emoción, comprensión y acción.

Sin duda, como lo afirma Costa (2010), refiriéndose a la obra de Aubenque (2010, p.9): "No hay nada más actual en el pensamiento de los filósofos de la Antigüedad griega que su propio, singular y específico modo de ser antiguos". Los modos de aprensión de la ética en el mundo griego helénico, nos puede ayudar a comprender las maneras en que la literacidad se entrelaza con nuestras formas de construir el mundo social y el sentido de la eticidad.

La integración del gimnasio a la polis griega, y particularmente a su centro cívico, el Ágora, marcó una significativa preocupación de los griegos clásicos por integrar la formación ciudadana como base estructural del currículo escolar.

Los gimnasios, según León Alonso (2008), estaban dedicados al cultivo del cuerpo, y a los objetivos de la preparación militar para defender la ciudad-estado. Hacia el final del siglo IV, los gimnasios se convirtieron en centros culturales e intelectuales donde concurrían jóvenes, invitados al cultivo de una vida examinada, digna de vivirse, como lo planteaba Sócrates; y por otra parte, se preparaban para la defensa de los recursos y los acervos culturales de la polis.

En sus amplios espacios abiertos, sus exaedras, estancias o salas, y sus jardines se fomentaba el diálogo, la argumentación y la discusión como fuentes de literacidad centrales de la formación en la Grecia antigua. Esta intención pedagógica que no solo involucraba la adecuada infraestructura escolar, sino un currículo organizado facilitaba el cultivo de los ciudadanos para y en la polis. Uno de los gimnasios más célebres en la Grecia helénica por sus implicaciones filosóficas de esa época hasta nuestros días, es la Academia (Akademeia), nombre que celebraba a un héroe local llamado Hekademos o Academo, en cuyos poblados bosques Platón fundó su propio gimnasio.

En la Academia de Platón se estudiaban todas las disciplinas contenidas en grandes conjuntos temáticos matemático-geométricos y filosófico-humanísticos. Allí el florecimiento del pensamiento crítico no solo pasaba por los discursos referidos a las matemáticas, o la música, sino también a la vida armónica y ética en la polis.

En las exaedras de los gimnasios y aun de las palestras (estructuras contiguas a los gimnasios, mayormente dedicadas al cultivo del cuerpo), se reunían a dialogar maestros, filósofos, y retores, acompañados por un foro de adolescentes interesados en las sesiones magistrales. Este escenario constituye una evolución importante en la presencia de recursos y fuentes de literacidad en la ephebeia (escuela para adolescentes), donde se cultivaba a los jóvenes para la participación en la vida política.

Por su parte, en el período de la Alta Edad Media, la enseñanza del Trivium (gramática, dialéctica y retórica) y el Quadrivium (aritmética, geometría, astronomía y música) en las escuelas monásticas y catedralicias en Europa, vinculaba una integración curricular que fundamentaba el cultivo del cuerpo y el espíritu. Aspecto que va desdibujándose paulatinamente en el inicio de las escuelas del renacimiento con el florecimiento del racionalismo. Para el siglo XIX, con la imposición de la enseñanza científica fundamentada por el positivismo, se establece una clara escisión entre el cultivo del espíritu y de la razón. 
En el primer cuarto del siglo XIX se acuña en Inglaterra el término de las 3Rs, referido a la adquisición de conocimientos en la escritura, la lectura y la aritmética (wRiting, Reading, and aRithmetic). En este triángulo de conocimientos se excluye la retórica, y la dialogicidad, fundamentos de la antigua ephebeia. La inclusión de la enseñanza de la aritmética al currículo público es tardía (inicios del siglo XX), puesto que antes, dicha instrucción particular, debía ser pagada por los padres de estudiantes de las escuelas públicas. La escisión entre el desarrollo de las 3Rs y la retórica y dialéctica griega como principales fuentes de la dialogicidad, generó una importante disminución de la capacidad de argumentación de los estudiantes en las escuelas modernas del siglo XX, convirtiendo a la instrucción en el centro de las preocupaciones escolares.

A mediados de los años 70 del siglo pasado, en América Latina, los trabajos de Freire (1970) referidos a la educación popular en los estados pobres del norte de Brasil, recuperan el sentido de la dialogicidad como fundamento de la agencia social y del desarrollo de una conciencia del papel de la educación en la construcción de la ciudadanía. El movimiento de Alfabetización iniciado por Freire en el Brasil, sienta las bases del concepto contemporáneo de la literacidad, con un claro componente de conciencia social, de posicionamiento político y del desarrollo de la subjetividad e intersubjetividad de los estudiantes.

El proceso de conocimiento se da a partir del análisis crítico del contexto donde el educando y el educador se encuentran. En el caos de la Alfabetización, eso comienza con la comprensión del lenguaje. Entonces el programa de alfabetización debe provenir del pueblo que se va a alfabetizar, no de los educadores que van a alfabetizarlo. Debe partir del análisis del lenguaje común que habla ese grupo. Por eso defiendo la investigación del universo léxico mínimo del pueblo (1998, p.55).

Denominamos recursos de literacidad aquellos objetos, espacios físicos y mediaciones que permiten generar ambientes de literacidad. Ellos representan la infraestructura instrumental, técnica y tecnológica de literacidad. Por su parte, las fuentes de literacidad son aquellas situaciones intencionales de aprendizaje significativo mediadas por el lenguaje, que se sirven de recursos de literacidad capaces para promover, de manera continua, la construcción de la subjetividad, la intersubjetividad y el sentido de una comunidad críticopolítica, orientada al ejercicio de una vida digna y ética. Hoy en día podemos evidenciar un significativo déficit de recursos y fuentes de literacidad que no permiten la vinculación del pensamiento crítico, los desarrollos de la ética, y el examen de una vida, como elementos consustanciales del currículo escolar moderno y contemporáneo.

Para terminar esta sección, en la interacción de la sophia y phrónesis se construye el espacio para el estudio de las experiencias, las emociones y la acción, componentes fundamentales de la Literacidad. La unión de la sophia-phrónesis, constituye una suerte de literacidad crítica que conjunta la experiencia sensible, la responsabilidad social, el mundo de la vida y el espacio de dialogicidad, como componentes sustanciales de una formación ética, justa y responsable.

La sophía se definió en primera instancia como "alguien entendido en algo", el desarrollo de las manualidades, el arte o manejo de una técnica, el gobierno de las ciudades. Es un tipo de modo de ser que conduce a la humanidad a ser artistas, artífices o tener un tipo de sabiduría (Zubiri, 1994). Se trata de un conocimiento acerca de los fines de la existencia, el examen del mundo independiente de las acciones humanas. 
De acuerdo con Zubiri (1944), la sophía, como teoría (episteme, racionalidad o sabiduría), instituye un aporte fundamental de la Grecia helénica que impactó, de manera significativa, el modo mental de situarse ante las cosas, más que en el lugar de los objetos sobre la cual recae el razonamiento. Igualmente, significa dentro de la epistemología, un conocimiento en tanto creencia justificada como verdad. Como afirmaba Platón, la epistéme se diferencia del concepto de doxa, vinculado mayormente a la creencia popular.

Por su parte, la phrónesis es definida por Gadamer (1972), como la virtud más alta del saber cuyo cometido es la orientación del obrar y de la vida humana, cuyo origen aristotélico vincula el concepto de sabiduría práctica. En este punto, la praxis no se refiere a la evidencia que se tiene de la construcción de un producto particular encaminado al éxito, sino en un saber que se instaura en la acción del individuo, cuya prueba es la calidad de la acción misma (Costa, 2010). La phrónesis significa para Aristóteles la areté (en griego), o virtus (virtud en latín), es decir, el cumplimiento íntegro y ético de la praxis.

La phrónesis encarna una disposición práctica de la acción ética que es consciente de los límites y oportunidades de cada situación de la falible naturaleza humana. De allí que Aubenque (2010, p.88) enuncie que, "el dominio de la prudencia, no es el Bien y el Mal en general, o el Bien y el Mal absolutos, sino el bien y el mal para el hombre".

En síntesis, la phrónesis es una conciencia de la acción humana que requiere habilidades para discernir cómo o por qué actuar virtuosamente, tomar decisiones que no afecten a otros de manera negativa y fomentar la areté o virtud en la práctica, la excelencia del carácter y la conciencia plena del actuar.

La literacidad en su sentido más ilustrativo (sophía), nos familiariza con el universo de conocimiento al que podemos y tal vez, podremos acceder, mientras que en su sentido más práctico, (phrónesis), se acerca tanto a la conciencia como a la coherencia de nuestras acciones y capacidades humanas. La literacidad desde estos referentes no se presenta como una trayectoria preestablecida o un fin vinculado al ritual de la banalidad del éxito, sino como trayectos de aprendizaje social, de construcción del conocimiento en comunidad y de la conciencia de la acción humana, donde las emociones tienen un papel predominante.

A este respecto, y dentro del campo de la filosofía política, Nussbaum (2008, p.45) afirma que, "las emociones encierran siempre una valoración o una evaluación, ya que llevan consigo la combinación del pensamiento sobre un objeto y el pensamiento sobre la relevancia o importancia de dicho objeto".

El estrecho vínculo que se teje entre las emociones y sus objetos, evidencia que las primeras no se relacionan sólo con sensaciones corporales, sino con una conexión mediada por la cultura, los imaginarios, las creencias, el racionamiento y juicio que establecemos alrededor de ellos. La valoración que hacen los sujetos del mundo con relación a su bienestar representan los propósitos y proyectos más relevantes de los individuos, puesto que se involucra un sentimiento donde su propio ser está implicado (Nussbaum, 2008). Este aspecto eudaimonístico proveniente de la ética de Aristóteles y personifica el ideal de felicidad humana.

De allí que Nussbaum (2008, p.177) afirme que, "en una criatura ética y socio-política, las propias emociones son éticas y socio-políticas, componentes de la respuesta a las 
preguntas ¿Sobre qué vale la pena interesarse?, y ¿Cómo he de vivir?”. Las emociones, en la visión neoestoicista de Nussbaum, simbolizan una forma de juicio mediante la cual brindamos valor y relevancia a determinadas cosas y personas. A razón de que las emociones son esenciales para el ejercicio de nuestra naturaleza, no es posible ignorarlas, pero sí orientarlas a una intencionalidad pedagógica a través de diferentes recursos y fuentes de literacidad. Tal vez, sea esto, el fundamento de una sabiduría práctica, ya enunciada por Aristóteles.

Elser (2001) asevera que cuando una emoción es desencadenada por un elemento sociocognitivo, representa la liberación de dicha emoción por una creencia relacionada con otros o con uno mismo, con el presente, el pasado o el futuro. De este modo podríamos considerar la estrecha relación que guardan las creencias, los juicios y las emociones. Al transformarse la creencia, cambia el juicio y, por tanto, también la emoción. Según López Carrascal (2016, p.91), "El carácter central de las creencias en las emociones se aprecia al percatarnos de cómo afectando las primeras se afectan las segundas [...]. Revelar el poder político de las emociones es justamente uno de los propósitos de la Retórica”.

Las emociones políticas a las que se refiere Nussbaum (2008) están encaminadas a promover la comprensión de la acción realizada mediante el juicio, e igualmente, a la reorientación de prácticas, como centro de toda educación ciudadana y, por tanto, de toda literacidad que apunte a la constitución política de las comunidades de aprendizaje.

Cuando nos encontramos en una sociedad, si no hemos aprendido a concebir nuestra persona y la de los otros de ese modo, imaginando mutuamente las facultades internas del pensamiento y la emoción, la democracia estará destinada al fracaso, pues ésta se basa en el respeto y el interés por el otro, que a su vez se fundan en la capacidad de ver a los demás como seres humanos, no como meros objetos (Nussbaum, 2010, p.25).

\subsection{El cultivo de la Humanidad: tensiones entre Ética y la Literacidad en la Educación Superior}

En el ámbito de la educación, los recursos y fuentes de literacidad son necesarios para movilizar capacidades, habilidades y saberes socio-cognitivos desde el inicio de la adquisición de la lengua, el desarrollo lógico-matemático y el cultivo de la ética y la política. Las instituciones educativas requieren vincular en su desarrollo curricular y de las prácticas educativas, una sabiduría práctica que remplace la formación basada en el modelo de competencias encaminadas al desempeño y al éxito escolar y profesional, hacia experiencias significativas de una vida digna y ética y a un pensamiento crítico, político y autónomo de los individuos de todas las sociedades.

La erosión de la excelencia -definida por Aristóteles como un modo de vivir una vida digna-, por la valoración cuantitativa acerca del desempeño (performance educativo), sumada a una concepción positivista de la formación, aparecen como síntomas constitutivos de la comercialización de las instituciones educativas, al embate de la globalización de los mercados de conocimiento y del Capitalismo Académico (Slaughter y Leslie, 1999; Slaughter y Rhoades, 2004; Correa Arias, 2011).

Esta hegemonía ideológica y política global sobre la función de la educación es legitimada en los escenarios económicos del Tratado de Lisboa, el Proceso de Bolonia y su instrumentación en el Proyecto Tunning, iniciado en el eje centro-europeo y difundido a nivel mundial. La mercantilización de la educación genera una deformación del mundo de la vida, 
de la experiencia, la emoción y la acción social que pone en peligro la construcción de la comunidad crítico-política y el establecimiento de un lazo social auténtico y ético.

Nussbaum (2010) identifica claramente en el libro, "Sin fines de lucro. Por qué la democracia necesita de las Humanidades", el mismo giro positivista que los trabajos de Slaughter y Lesli (1999), Slaughter y Rhoades (2004), Colado (2005) y Correa Arias (2011), entre otros, denuncian cómo la instauración de un régimen de producción de bienes de capital intelectual en la universidad contemporánea la obligan a participar en el mercado global educativo del prestigio y la calidad educativa.

En medio de estos campeones de los rankings se encuentran las denuncias del cierre de facultades de humanidades y el despido de filósofos, sociólogos y humanistas. Como anota Wright $(2014,2015)$, se trata de la mercantilización de la universidad contemporánea y de la corrupción de altos funcionarios en instituciones universitarias, aún en aquellas consideradas como instituciones educativas de primer mundo.

La ausencia de las humanidades en el tejido curricular, a lo largo de todos los programas académicos y en todas las disciplinas y cursos de la universidad profundiza la escisión entre la construcción de saberes técnicos y el mundo de la vida de los educandos. Este abandono institucional lacera valores y capacidades sociales fundamentales para la construcción de la propia comunidad y produce una gran contradicción entre los discursos oficiales de la institución y los intereses propios de los sujetos. También limita los recursos y fuentes de literacidad crítico necesarios para el desarrollo de un pensamiento crítico, ético y autónomo de los estudiantes.

Se identifica pues, un déficit de literacidad en las instituciones educativas al pretender dar respuestas a las preguntas que se construyen en cada contexto de la acción social, mediante el cultivo único de las habilidades cognitivas. Este abandono institucional representado en la escisión entre literacidad y ética, no sólo se observa en el campo de la experiencia cotidiana, sino también en los procesos de argumentación. Vargas y Cárdenas (2005, p.136) plantean que,

por igual se tiene que formar en la argumentación los sectores y miembros de las diferentes versiones políticas, como las diversas profesiones en la sociedad. De ahí que se vea previsible que la retórica se llegue a imponer, tarde o temprano, como campo de formación, y sobre todo, como campo específico del ejercicio de formación en las ciencias. En este sentido, es tan relevante que una persona aprenda a deconstruir como a construir la argumentación, pues con ello queda habilita para ejercer ciudadanía, para contribuir a hacer valer sus intereses en la vida pública.

\section{Método}

La investigación se propone analizar las percepciones de los estudiantes del centro universitario de Ciencias Económico-administrativas (CUCEA) de la Universidad de Guadalajara, en México, con respecto al valor y las implicaciones que los cursos de Ética Profesional tienen en la vida universitaria, y la proyección de dichos aprendizajes aplicables en la vida profesional, con el fin de identificar los obstáculos y oportunidades institucionales al acceso a recursos y fuentes de literacidad orientadas a la construcción de una ciudadanía responsable. Debido a que en el currículo de pregrado concretamente en el tronco común de toda la universidad se ofrece el curso de Ética Profesional, 160 mil estudiantes de los casi 300 
mil estudiantes que conforman la población estudiantil de esta casa de estudios, están obligados a inscribirse en este curso.

La investigación se posicionó en un paradigma socio-crítico y un sub-paradigma éticoformativo-exploratorio, desde donde se analizaron los relatos de los sujetos permitiendo la emergencia de categorías, capacidades y habilidades sociales, tales como la moral, los rituales de violencia simbólica institucional, la vergüenza, el silencio, la indiferencia, la corrupción institucional, etc., e igualmente, los principales aspectos que limitan una vida ética y digna.

Se utilizó una metodología cualitativa de corte etnográfico. Se trata de un caso de estudio de tipo intrínseco referido a los estudiantes de los cursos de Ética Profesional del Centro Universitario de Ciencias Económico-administrativas (CUCEA), de la Universidad de Guadalajara, México.

En la investigación se realizaron entrevistas semi-estructuradas y grupos focales, además del análisis documental de los trabajos de los estudiantes del curso de Ética Profesional en una muestra de ocho (8) grupos durante el período académico, 2014A a 2017B. Se utilizó el software Atlas.ti, para la organización y sistematización de los datos obtenidos; y como técnica de análisis de la información, el Análisis Crítico de Discurso.

\section{Resultados}

Los datos encontrados se vinculan a tres lugares de análisis:

1) los trabajos de los estudiantes de este curso con respecto a la importancia de fuentes de literacidad que les brinda el curso de Ética Profesional;

2) ocho (8) entrevistas a profundidad con el mismo propósito; y

3) cuatro (4) grupos focales.

Además a cuatro (4) aspectos nucleares:

1) Las percepciones de los estudiantes acerca de los cursos de ética.

2) la calidad de los recursos y fuentes de literacidad y el desarrollo de capacidades y habilidades socio-cognitivas a través de visitas a comunidades desfavorecidas.

3) La afiliación como elemento sustancial para la constitución de las identidades plurales.

4) La construcción de la intersubjetividad y la subjetividad.

En el primer aparte se concentran las percepciones de los estudiantes acerca de la estructura, diseño, desarrollo curricular y didáctico, las aplicaciones, e implicaciones del curso de ética, e igualmente, su relación con resto del tejido curricular del curso de Ética Profesional del CUCEA, Universidad de Guadalajara. Se presentan algunos testimonios, entre las ocho (8) entrevistas que se realizaron y, luego, estos datos totales se analizan en una tabla que evidencia las categorías emergentes.

El curso tiene una estructura muy pertinente, apropiada, es uno de los mejores cursos. No comprendo por qué la universidad no le da más importancia. Cuando me salgo de esta clase y sigo con otra, me siento sola, desconectada porque volvemos a la misma rutina, y a ciertas tiranías de los docentes. Nos dicen que seamos amigos de los docentes, pero es simulación para que no nos 
vaya mal. El curso de ética es un respiro, se tocan cosas que son de vital importancia para la vida de nosotros (María, 2017).

En la cita se puede observar la desconexión entre el curso de Ética Profesional, como espacio, recurso y fuente de literacidad para el desarrollo de la subjetividad y la intersubjetividad y los demás cursos, cuyos contenidos programáticos no vinculan aspectos éticos.

La tabla 1 muestra la percepción de los estudiantes del CUCEA, acerca de los ítems y lugares de análisis mencionados.

Tabla N.1. Percepciones de los estudiantes Ética Profesional, CUCEA. 2014B-2017A.

\begin{tabular}{|c|c|c|}
\hline \multirow[t]{2}{*}{ Diseño Curricular } & \multirow[t]{2}{*}{ Ámbito Didáctico } & $\begin{array}{l}\text { Relaciones del programa de curso con el } \\
\text { CUCEA. Consistencia }\end{array}$ \\
\hline & & Inconsistencias \\
\hline \multirow[t]{2}{*}{$\begin{array}{l}\text { 1. Contenidos de } \\
\text { interés y } \\
\text { vigencia. }\end{array}$} & \multirow{2}{*}{$\begin{array}{l}\text { Temas que se ajustan en } \\
\text { un gran porcentaje a los } \\
\text { intereses de los } \\
\text { estudiantes y con mucha } \\
\text { aplicación en la vida de } \\
\text { los estudiantes }\end{array}$} & $\begin{array}{l}\text { Fuerte cohesión con temas actuales. } \\
\text { Invitación al compromiso, armonía entre } \\
\text { teoría y práctica. }\end{array}$ \\
\hline & & $\begin{array}{l}\text { Temáticas de gran interés para el curso, pero } \\
\text { poca o escasa para el resto de los docentes y } \\
\text { estudiantes en los demás cursos del CUCEA. } \\
\text { En su gran mayoría solo se hablan estos } \\
\text { temas a profundidad en la clase de ética. }\end{array}$ \\
\hline \multirow[t]{2}{*}{$\begin{array}{l}\text { 2. Coherencia } \\
\text { temática }\end{array}$} & \multirow{2}{*}{$\begin{array}{lrr}\text { Actividades } & \text { que } \\
\text { corresponden a } & \text { las } \\
\text { temáticas y } & \text { se } \\
\text { complementan con visitas } \\
\text { a instituciones }\end{array}$} & $\begin{array}{l}\text { Los cursos tienen un ordenamiento temático } \\
\text { lógico. }\end{array}$ \\
\hline & & $\begin{array}{l}\text { La universidad no se interesa por generar más } \\
\text { actividades con respecto a los temas del } \\
\text { curso. }\end{array}$ \\
\hline \multirow[t]{2}{*}{$\begin{array}{l}\text { 3. Secuencia de los } \\
\text { temas }\end{array}$} & \multirow{2}{*}{$\begin{array}{l}\text { Conocimientos se van } \\
\text { construyendo en la } \\
\text { medida que se están } \\
\text { desarrollando los temas. }\end{array}$} & $\begin{array}{l}\text { La secuencia es lógica y sencilla, se } \\
\text { comprende el encadenamiento de los temas. }\end{array}$ \\
\hline & & $\begin{array}{l}\text { El curso debería permanecer en todos los } \\
\text { semestres. }\end{array}$ \\
\hline \multirow[t]{2}{*}{ 4. Objetivos claros } & \multirow{2}{*}{$\begin{array}{l}\text { Los objetivos responden } \\
\text { en buena medida a las } \\
\text { expectativas de } \\
\text { estudiantes. }\end{array}$} & $\begin{array}{l}\text { Los objetivos están diseñados para formar } \\
\text { personas con valores. }\end{array}$ \\
\hline & & $\begin{array}{l}\text { Fuera de clase las personas no se } \\
\text { comprometen a actuar de manera ética. La } \\
\text { institución se contradice. Indiferencia, } \\
\text { corrupción en estudiantes, docentes y } \\
\text { administrativos y los objetivos no se cumplen } \\
\text { sino en clase. }\end{array}$ \\
\hline \multirow[t]{2}{*}{$\begin{array}{l}\text { 5. .Metodología } \\
\text { apropiada }\end{array}$} & \multirow[t]{2}{*}{$\begin{array}{lcc}\text { Metodología permite la } \\
\text { motivación } & \text { de } & \text { los } \\
\text { estudiantes. } & & \end{array}$} & $\begin{array}{l}\text { Las actividades permiten un contacto directo } \\
\text { con la realidad. Clases y visitas son un } \\
\text { excelente complemento. }\end{array}$ \\
\hline & & $\begin{array}{l}\text { Desmotivación por lo que se hace en clase y } \\
\text { en las instituciones y lo que se vive en el } \\
\text { CUCEA. }\end{array}$ \\
\hline \multirow{2}{*}{$\begin{array}{l}\text { 6. Implicaciones } \\
\text { del curso en la } \\
\text { formación del } \\
\text { estudiante }\end{array}$} & \multirow[t]{2}{*}{$\begin{array}{l}\text { Conocer la teoría con la } \\
\text { práctica, y viceversa. }\end{array}$} & $\begin{array}{l}\text { Muy importantes por la amistad, los valores y } \\
\text { la motivación que se fomenta. }\end{array}$ \\
\hline & & $\begin{array}{l}\text { Profesores tiranos, poco a la participación, mal } \\
\text { trato, control y poder. }\end{array}$ \\
\hline
\end{tabular}

Fuente: Construcción Propia, 2018. 
En la tabla 2 se puede ver la correlación entre las visitas institucionales y el aporte de fuentes de calidad y las capacidades y habilidades socio-cognitivas que estas aportan.

Tabla 2. Percepción de los estudiantes del CUCEA. Período 2014B-2017A.

\begin{tabular}{|c|c|c|c|}
\hline $\begin{array}{l}\text { Visitas a } \\
\text { instituciones como } \\
\text { proveedoras de } \\
\text { fuentes de } \\
\text { Literacidad }\end{array}$ & $\begin{array}{l}\text { Fuentes de } \\
\text { Literacidad } \\
\text { Categorías de } \\
\text { Análisis }\end{array}$ & \multicolumn{2}{|l|}{ Capacidades } \\
\hline \multirow[t]{9}{*}{$\begin{array}{l}\text { Instituciones de } \\
\text { atención a personas } \\
\text { en situación } \\
\text { desfavorable: } \\
\text { Centros de salud, } \\
\text { casas de asistencia, } \\
\text { asilos para } \\
\text { ancianos, etc. }\end{array}$} & \multirow[t]{3}{*}{ Emocionales* } & Filiación & $\begin{array}{l}\text { Amor, admiración- } \\
\text { gusto, satisfacción vía, } \\
\text { delicadeza, estima, generosidad, } \\
\text { honestidad perdón, ternura, } \\
\text { fortaleza, hospitalidad, diálogo, } \\
\text { sentido, sinceridad, respeto, } \\
\text { reconocimiento- }\end{array}$ \\
\hline & & Cognitivas & $\begin{array}{ll}\text { Aprendizaje, } & \text { atención, } \\
\text { comprensión } & \text { juicio, } \\
\text { asombro } & \text { reflexión }\end{array}$ \\
\hline & & Reacción & $\begin{array}{l}\text { Asco, ira, lástima, miedo, } \\
\text { tristeza, nostalgia. }\end{array}$ \\
\hline & \multirow{2}{*}{\begin{tabular}{|l|} 
Construcción \\
Subjetiva e \\
Intersubjetiva**
\end{tabular}} & Agencia & $\begin{array}{l}\text { Servicio, compromiso, } \\
\text { responsabilidad, liderazgo, }\end{array}$ \\
\hline & & Sociales & $\begin{array}{l}\text { Autonomía, valoración, } \\
\text { imaginación, sensibilización , } \\
\text { cuidado, confianza, gratitud, } \\
\text { compasión, adaptación, pasión. }\end{array}$ \\
\hline & \multirow[t]{2}{*}{$\begin{array}{l}\text { Afiliación y } \\
\text { pertenencia*** }\end{array}$} & Motivacionales & $\begin{array}{l}\text { Acceso lazos de confianza amista } \\
\text { apoyo, compromiso, convivenci } \\
\text { inclusión, implicación, inserció } \\
\text { social, valores, integració } \\
\text { compañía. }\end{array}$ \\
\hline & & Deprivadores & Abandono, carencia, rechazo. \\
\hline & \multirow[t]{2}{*}{ Prospectivas**** } & $\begin{array}{l}\text { Proyecto } \\
\text { personal }\end{array}$ & $\begin{array}{l}\text { Calidad de vida, plenitud, } \\
\text { felicidad, formación, éxito, } \\
\text { decisión y desarrollo personal, } \\
\text { vida digna, oportunidades, }\end{array}$ \\
\hline & & $\begin{array}{l}\text { Proyecto } \\
\text { institucional/co } \\
\text { munitario }\end{array}$ & Familia, socialización, empleo. \\
\hline
\end{tabular}

Fuente: Construcción propia, 2018. *Concepción de las emociones desde la sociología de la experiencia (Scribano, 2012). **Reconocimiento de las personas sobre la base de la justicia social (Taylor, 1993). ***Construcción identitaria y la afiliación. Filosofía política (Sen, 2006). ****Construcción de un proyecto personal o colectivo. (Ricoeur, 2006).

En la siguiente cita se observa la percepción de un estudiante participante en las visitas institucionales, al momento de la retroalimentación en su trabajo final. La visita fue realizada en un centro de rehabilitación de niños con cáncer ubicado en el Municipio de Guadalajara, México.

Yo iba con la mentalidad de dar fortaleza a los niños, pero al final, yo salí fortalecido. Los niños nos dan la fuerza y provocan el cambio; hay que aprender de ellos y de sus papás. Es hermoso compartir tiempo con estos angelitos y ver esas sonrisas sinceras llenas de agradecimiento, [ellos] nos dan 
gran lección de vida. La mejor manera de sentirte mejor y ayudar a los demás. Al estar ahí te pones en el papel de la gente con discapacidad y es muy difícil, tienes que tener muchas ganas de salir adelante y fuerza mental para poder lograr todo los que ellos consiguen a diario. Fue una experiencia maravillosa, cambió mi perspectiva por completo, tengo una gran admiración por las personas que trabajan y que están en estos Centros (Antonio, 2017).

\section{Discusión}

Dentro del primer eje de análisis se puede inferir de los resultados de la tabla 1 que:

a) existe una buena correlación del diseño, desarrollo curricular y la metodología de los ocho (8) cursos de Ética Profesional analizados. Los estudiantes anotan que debería aumentarse las visitas en las instituciones, por el valor de la experiencia directa con los sujetos.

b) existe una evidente desconexión entre los cursos de ética y los demás cursos de este centro universitario. No se conservan los mismos valores, y se evidencia violencia en la formación, con profesores son poco éticos $\mathrm{y}$, no en pocas ocasiones, un trato irrespetuoso y arbitrario del docente hacia los estudiantes.

c) los estudiantes denuncian que son tomados en cuenta y reconocidos en clase de ética, pero luego, categorías sociales como la participación, la honestidad, la hospitalidad desaparecen en la relación con otros docentes y otros cursos.

d) por fuera de los cursos de ética, es relevante la presencia de la vergüenza ante los otros y reina la desconfianza y la falta de compañerismo.

Adam Smith (1790) afirma que existe un sentimiento de vergüenza, de culpa moral cuando no se ha prestado atención a la voz de la conciencia del espectador interno, o que existe una clara sanción social de orden moral, cuando el sujeto se adhiere completamente a la opinión del espectador externo. En Smith el espectador tanto interno como externo se forma en el seno de la comunidad a través de la comunicación afectiva y efectiva con los demás, lo que lo hace imparcial, pero igualmente, hospitalario. De allí, que la falta de hospitalidad y reconocimiento social profundizan la vergüenza social.

Dentro del segundo eje de análisis, se puede deducir de la información de la Tabla N.2, así como a la cita que le continúa, que los recursos y las fuentes de literacidad ofrecidas en el curso de "Ética Profesional" y en las visitas a comunidades desfavorecidas, sirvieron para traer al consciente, emociones políticas en los estudiantes, que superaban las sensaciones corporales iniciales. Estas emociones que se desplazan hacia los otros, constituyen, según Nussbaum (2014), el enlace entre la simpatía-empática, elemento fundamental del desarrollo de la ética.

Como lo afirma Habermas (2003),

solo como participantes de un diálogo inclusivo y orientado hacia el consenso se requiere de nosotros que ejerzamos la virtud cognitiva de la empatía hacia las diferencias con los otros en la percepción de una situación común. Se supone que debemos interesarnos cómo procederían cada uno de los demás participantes desde su propia perspectiva, para la universalización de todos los intereses implicados. (Habermas, 2003, pp.23-24). 
Lo anterior, nos permite afirmar que los estudiantes, gracias a la disponibilidad de recursos de literacidad como las discusiones, las lecturas, los videos y las fuentes de literacidad representadas en la dialogicidad, la interiorización, la hospitalidad, la comprensión en comunidad y la simpatía-empática que brindaron conjuntamente el curso y las visitas, promueven el desarrollo de un sentido ético situado y real acerca de la fragilidad y las capacidades humanas en ambientes sociales desfavorecidos. De allí que sea justo afirmar con Nussbaum (2014, p.15), que "Todas las sociedades, tienen que pensar en sentimientos como la compasión ante la pérdida, la indignación ante la injusticia o la limitación de la envidia y el asco en aras de una simpatía inclusiva".

Gracias a estas visitas se promovieron, como se aprecia en la tabla 2, el desarrollo de capacidades sociales (Sen, 1993; Nussbaum, 2011), como la filiación (afecto), el respeto, la afiliación (pertenencia), hospitalidad y la compasión, a parte de las habilidades cognitivas. Gracias a la experiencia sensible con sujetos que tiene una condición diferente a la suya, se empiezan a delinear en la personalidad estas capacidades en forma de deseo y proyecto. De un proyecto individual y un proyecto profesional y comunitario.

Yo volvería a ir, nunca pensé que terminaría gustándome tanto volver a visitar un lugar que veía como deprimente y repulsivo. Ahora veo las cosas muy distintas. Estas personas son seres humanos y requieren de acompañamiento (Jesús, 2018)

Los estudiantes del curso pudieron llegar a ser conscientes de capacidades que, en alguna medida, estaban ausentes en ellos y presentes en sujetos que poseen una condición menos ventajosa (discapacidad, enfermedades, vejez, condiciones de desarrollo cognitivo, etc.), tales como la resiliencia, la tolerancia, la hospitalidad, entre otros.

El tercer eje del análisis se refiere a la afiliación un elemento sustancial para la constitución de las identidades plurales (Sen, 2006) y para la edificación de la comunidad. El sentido de la comunidad en este nivel plantea el reconocimiento mutuo en instituciones justas (Ricoeur, 2006). Se trata de combatir un reconocimiento ideológico en las instituciones que fallan en concretar en el mundo material un comportamiento de reconocimiento continuo y responsable (Honneth, 2006) y de ser sensibles y compasivos frente a personas en situaciones desfavorables y en general, al otro como ser humano falible y capaz.

Finalmente, el cuarto aspecto de análisis se traduce en el proyecto social que anuda el compromiso más importante de la vida ética: la construcción de la intersubjetividad y la subjetividad, que parte de emociones básicas y logra evolucionar a emociones políticas, permitiendo la afiliación y la pertenencia necesaria para construir una comunidad orientada al ejercicio de una vida digna.

El mayor aporte a la construcción de una vida ética de los estudiantes de instituciones de educación es la generación de fuentes de literacidad de calidad que permitan el desarrollo y alcance de una vida digna por fuera de un sistema de consumismo salvaje sin sentido. La provisión de recursos y fuentes de literacidad de calidad orientadas a la ciudadanía, debería ser una de las principales responsabilidades de los sistemas e instituciones educativas. Estas fuentes proveen a los estudiantes de emociones primarias y políticas, implicándolos con el mundo de la vida. Sirven a su vez de conectores con la experiencia de otros mundos de vida que no son los propios y que aun, en este ecosistema planetario compartimos, mayormente, bajo el régimen de la inconciencia y la indiferencia. 
Los cursos de "Ética Profesional" que asumen la responsabilidad y consciencia de fomentar procesos de subjetividad e intersubjetividad plantean dentro de sus objetivos, sobrepasar la instrumental tarea de producir futuros gerentes, empresarios o profesionistas, para brindar recursos y fuentes de literacidad que promuevan la auto-realización de los sujetos. La universidad no puede ser indolente ante las incoherencias de sus currículos desconectados de la realidad social que nos rodea. Mientras los mensajes de las instituciones de educación superior promulgan la autonomía de las personas, en su interior ocurren las mayores contradicciones que se alejan de toda propuesta ética.

A su vez, mientras las instituciones de educación superior continúen tratando la ética como un contenido curricular particular y no como un proyecto formativo transversal en el cual toda la comunidad universitaria y la sociedad estén implicadas y comprometidas no dejará de ser un curso optativo alejado de la realidad universitaria y del contexto social, útil para justificar el cumplimiento de una responsabilidad olvidada por estas instituciones educativas.

La literacidad y la ética aparecen juntas e igualmente divididas en el horizonte contemporáneo de la educación universitaria, tal como cuando observamos el cielo y el mar unidos en un amanecer o en un ocaso en la playa; sin embargo, es en este horizonte que la intersección de la literacidad y la ética sean parte de la responsabilidad de la universidad de construir su proyecto cosmopolita de inclusión social y de formación política, conducente al alcance y ejercicio de una vida digna de cada miembro de su comunidad educativa, para que estos sirvan como embajadores de la autonomía y la construcción democrática de la sociedad y de sus instituciones.

\section{Referencias bibliográficas}

Aubenque, P. (2010). La prudencia en Aristóteles. Buenos Aires, Argentina: Las Cuarenta.

Correa Arias, C. (2011). Public Policies on Higher Education: Rhetoric and Grammar of Modernity and the Limits of Social Recognition. The International Journal of Interdisciplinary Social Sciences: Annual Review, 5(9), 177-190. doi:10.18848/18331882/cgp/v05i09/51858

Costa, I. (2010). Prólogo. En Pierre Aubenque. La prudencia en Aristóteles. Buenos Aires, Argentina: Las Cuarenta.

Elster, J. (2001). Sobre las pasiones. Emoción, adicción y conducta humana. Barcelona, España: Paidós.

Freire, P. (1998). Conversación libertaria con Edson Passetti. San Pablo, Brasil, Editora Imaginário.

Gauthier, Ph. (1995). Notes sur le role du gymnase dans les cités hellenístiques en M. Würrle, P. Zanker, Stadtbild und Blirgerbild im Hellenismus. Hamburg: Ed. Hobein.

Habermas, J. (1999). La inclusión del otro: Estudios de teoría política. Barcelona, España: Paidós Básica. 
Honneth, A. (2006). El reconocimiento como ideología. Isegoría, O(35), 129-150. doi:10.3989/isegoria.2006.i35.33

Ibarra, E, (2005). Origen de la empresarialización de la universidad: el pasado de la gestión de los negocios en el presente del manejo de la universidad, en Revista de la Educación Superior, 34(134), 13-37. Recuperado de https://bit.ly/2S3N7Yy

León Alonso, $\mathrm{M}^{\mathrm{a}} \mathrm{P}$. (2008). Los espacios del saber y del pensamiento en el mundo griego. Boletín de la Real Academia Sevillana de Buenas Letras: Minervae Baetica. 36, 33-80. Recuperado de https://bit.ly/2PWCPqs

Levinas, E. (1987). Totalidad e infinito. Salamanca: Sígueme.

López Carrascal, L. F. (2016). Las emociones como formas de implicación en el mundo. El caso de la ira. Estudios de Filosofía, 53. doi:10.17533/udea.ef.n53a05

Nussbaum, M. C. (2014). Emociones políticas. Por qué el amor es importante para la justicia social. México: Paidós.

Nussbaum, M. C. (2011). Creating Capabilities. The Human Development Approach. United States of America: Harvard University Press.

Nussbaum, M. C. (2010). Sin fines de lucro. Buenos Aires: Katz.

Nussbaum, M. C. (2008). Paisajes del pensamiento. Barcelona: Paidós.

Nussbaum, M.C. (2005). El cultivo de la humanidad. Una defensa clásica de la reforma en la educación liberal. México: Paidós Básica.

Ricoeur, P. (2006). Caminos del reconocimiento. Tres estudios. México: Fondo de cultura Económica.

Scribano, A, (2013). Sociología de los cuerpos/emociones. Revista Latinoamericana de Estudios sobre Cuerpos, Emociones y Sociedad. 10, 93-113. Recuperado de https://bit.ly/35Hx46N

Sen, A. (2006). Identity \& Violence. The illusion of Destiny. London: Penguin Books.

Sen, A. (1993) "Capability and Well-being", en M. Nussbaum y A. Sen (eds.) The Quality of Life. Oxford, Clarendon Press. (Traducción de R. Reyes, in M. C. Nussbaum y A. Sen (eds.) La calidad de vida. Tercera reimpresión. México: Fondo de Cultura Económica, 2002).

Shore, C., y Wright, S. (2015). Governing by numbers: audit culture, rankings and the new world order. Social Anthropology, 23(1), 22-28. doi:10.1111/1469-8676.12098

Smith, A. (1790). Theory of Moral Sentiments, or An Essay towards An Analysis of the Principles by which Men naturally judge concerning the Conduct and Character, first of their Neighbors, and afterwards of themselves, to which is added a Dissertation on the Origin of Languages. II (Sixth ed.). London: A. Strahan; and T. Cadell in the Strand; and T. Creech and J. Bell y Co.: Edinburgh. 
Literacidad y ética. Percepciones de estudiantes de educación superior sobre el curso

Slaughter S. y Larry L. (1997). Academic capitalism: politics, policies and the entrepreneurial university. Baltimore: Johns Hopkins.

Slaughter, S. y Rhoades, G. (2004). Academic Capitalism and the New Economy. Baltimore: The John Hopkins University Press.

Taylor, Ch. (1993). La política del reconocimiento. En El multiculturalismo y la política del reconocimiento. México: Fondo de Cultura Económica. Recuperado de https://bit.ly/34ypXvI

Vargas Guillen, G, y Cárdenas Mejía, L. G. (2005). Retórica, poética y formación. De las pasiones al entimema. Medellín: Universidad Antioquia, Universidad Pedagógica Nacional.

Wright, S. (2014). Knowledge that counts: point systems and the governance of Danish universities. En A. I. Griffith y D. E. Smith (Eds). Under New Public Management, (pp.294-338). Toronto: University of Toronto Press. doi:10.3138/9781442619463-014

Zubiri, X. (1944). La idea de filosofía en Aristóteles, en Naturaleza, Historia, Dios. Madrid: Editora Nacional. 
